\title{
Systematic review and meta-analysis: the value of MRI chemical-shift imaging in the evaluation of bone quality in patients with osteoporosis
}

\author{
Ye Wang, Hong Li, Xin Yang, Chao Zhang, Haiyang Xie \\ Department of Orthopedics, Affiliated Hospital of North Sichuan Medical College, Nanchong, China \\ Contributions: (I) Conception and design: Y Wang, H Xie; (II) Administrative support: H Li; (III) Provision of study materials or patients: Y Wang, \\ X Yang, C Zhang, H Xie; (IV) Collection and assembly of data: All authors; (V) Data analysis and interpretation: H Li, X Yang, C Zhang; (VI) \\ Manuscript writing: All authors; (VII) Final approval of manuscript: All authors. \\ Correspondence to: Haiyang Xie. Department of Orthopedics, Affiliated Hospital of North Sichuan Medical College, No. 63 Wenhua Road, Shunqing \\ District, Nanchong, China. Email: nsmcxiehaiyangnc@163.com.
}

\begin{abstract}
Background: Osteoporosis is a systemic skeletal disease with no obvious symptoms in its early stages. The decreases in bone density and bone mass are associated with the deterioration of bony microstructure in the body, due to a variety of reasons. The current assessment criteria for osteoporosis are still not perfect. MRI chemical-shift imaging shows potential to be a valuable technique in the bone quality assessment of osteoporotic patients.

Methods: Randomized controlled studies on osteoporosis assessment were searched for in Englishlanguage databases, including PubMed, Web of Science, Embase, and The Cochrane Library. Inclusion criteria were: (I) literature focused on the assessment of osteoporosis by chemical displacement imaging; (II) studies evaluating the bone quality of osteoporosis; (III) studies with at least 15 samples; (IV) studies concerned with the evaluation of osteoporosis by chemical shift encoded magnetic resonance imaging (CSE$\mathrm{MRI}$ ); and (V) studies with outcome indexes for bone mineral density (BMD), body mass index (BMI), and bone marrow fat fraction (BMFF). The Jadad tool was used to evaluate the quality of the included studies, and the Review Manager v. 5.3 software was used to conduct the meta-analysis of the experimental data.

Results: A total of 7 suitable articles were selected. According to the results of the meta-analysis, for the osteoporosis group and normal group: BMD: $\mathrm{MD}=41.91$ [95\% confidence interval (CI): 40.15 to 43.66], $\mathrm{Z}=46.82(\mathrm{P}<0.00001)$; $\mathrm{BMI}: \mathrm{MD}=-1.43$ (95\% CI: -2.02 to -0.84$), \mathrm{Z}=4.76(\mathrm{P}<0.00001)$; $\mathrm{BMFF}: \mathrm{MD}=-18.34$ (95\% CI: -20.32 to -16.36$), \mathrm{Z}=18.15(\mathrm{P}<0.00001)$.

Discussion: MRI chemical-shift imaging can effectively measure BMD and bone mass, including in osteoporotic patients. However, the outcome indicators included in this study are not sufficient to assess the utility of MRI chemical shift imaging for evaluating bone mass in osteoporotic patients, and further expansion of the sample size and outcome indicators is needed.
\end{abstract}

Keywords: Osteoporosis; chemical displacement imaging; bone mineral density (BMD); bone marrow fat fraction (BMFF); meta-analysis

Submitted Oct 31, 2021. Accepted for publication Dec 10, 2021.

doi: 10.21037/apm-21-3479

View this article at: https://dx.doi.org/10.21037/apm-21-3479

\section{Introduction}

Osteoporosis is a systemic metabolic bone disease $(1,2)$ with no obvious symptoms in the initial stages of disease. The causes of osteoporosis are complex and diverse. Generally, osteoporosis will lead to a decrease in the bone density and bone mass of the whole body, and the destruction of 
bone microstructure, including brittle fractures caused by minor trauma and body deformities resulting from vertebral compression fractures (3). Therefore, osteoporosis leads to a high rate of disability and mortality, imposing a severe psychological burden on patients and a severe economic burden on families. According to current data, about 200 million people are affected by osteoporosis, globally. In 2018, in China, $3.2 \%$ of people aged from 40 to 49 years and $6 \%$ of men and $32.1 \%$ of women aged over 50 years suffered from osteoporosis. The prevalence of osteoporosis in women over 65 years old is as high as $51.6 \%$. In 2016, when China's population was growing older at a faster rate than almost all other countries, the probability of people aged over 60 years suffering from osteoporosis was as high as $36 \%$. Fracture is a serious symptom of osteoporosis. In 2010, more than 2 million people in China suffered from fracture due to osteoporosis $(4,5)$. Therefore, finding ways to enhance the early diagnosis and prevention of osteoporosis is of paramount importance. Bone mass and bone density have been a focus in imaging studies of osteoporosis in clinical practice as bone mass is determined by detecting bone mineral density (BMD). Both are important indicators of osteoporosis (6), and also play important roles in the observation of the efficacy of drugs used to manage osteoporosis. Dual X-ray absorptiometry (DXA) $(7,8)$, which measures BMD, has long been regarded as the clinical standard for detecting and diagnosing osteoporosis, but its accuracy is lower than that of quantitative computed tomography (QCT) DXA $(7,8)$. Yet, the high radiation dose and high cost of QCT has limited its clinical application (9). Furthermore, because magnetic resonance imaging (MRI) signals from solid-state tissues such as bone are very low and do not show details of bone very well, MRI has not been generally applied to the measurement of BMD in clinical practice. Studies have shown, though, that MRI chemical-shift imaging methods can be very good at performing bone density measurements, by measuring the cone signal decline index to reflect the change of bone fat content, thus proving the value of MRI chemical-shift imaging in the diagnosis and evaluation of osteoporosis.

Advances in MRI hardware and software technology and the increased use of diffusion-weighted imaging (DWI) are slowly making it possible to index and classify osteoporosis. By studying the apparent diffusion coefficient (ADC) values in patients with lumbar vertebral body measurements, vertebral osteoporosis in patients can be evaluated to support early detection and prevention and, to a certain extent, save medical costs associated with a delayed diagnosis $(10,11)$. The magnetic resonance degree shift imaging is achieved with two echo techniques to achieve an image of the same opposite phase in one scan, which can be preliminary judgment on the amount of bone marrow fat content by observing the amount of fat signal on the reverse phase image. Magnetic resonance degree shifts more research in abdominal lesions and bone marrow system lesions. In recent years, MRI chemical-shift imaging has gained recognition as a useful tool in the assessment of osteoporosis based on the evaluation of vertebral bone marrow fat content; however, because studies on the application of MRI chemical-shift imaging in the assessment of osteoporosis have lacked large sample sizes and longterm follow-up, further research is required to support the theoretical basis for the clinical application of MRI chemical-shift imaging in the assessment of osteoporosis. Allon et al. [2018] (12) initially compared the value of computer tomography (CT), magnetic resonance imaging (MRI), X-ray absorption assay (DXA), and ultrasonic method in vulnerability fracture prediction, indicating that MRI pairs Quality omission is sensitive and specific, and CT can predict fracture occurs, and ultrasonic examination can predict fracture of fracture occurs, and DXA can be used as a screening tool for osteoporosis. In this study, the value of MRI chemical transplant imaging was analyzed in the osteoporosis patients.

In this article, the evaluation of osteoporosis by MRI chemical-shift imaging is analyzed and discussed in detail. The changes of BMD and bone marrow fat fraction (BMFF) in different patients were studied to understand the theoretical basis of the role of MRI chemical-shift imaging in the clinical prevention and monitoring of osteoporosis.

We present the following article in accordance with the PRISMA reporting checklist (available at https://dx.doi. org/10.21037/apm-21-3479).

\section{Methods}

\section{Literature search strategy}

English-language databases, including PubMed, Web of Science, Embase and The Cochrane Library, were systematically searched for articles published from January 2000 to September 2020, "chemical shift imaging", "osteoporosis", "MRI”, "water-fat", "assessment", "bone 
density", "bone mass", etc. were used as search terms.

\section{Inclusion and exclusion criteria for study selection}

Inclusion criteria for studies were: (I) focused on the assessment of osteoporosis by chemical-displacement imaging, in China and abroad; (II) directly or indirectly evaluated bone quality for osteoporosis; (III) included at least 15 samples; (IV) used CSE-MRI methods to evaluate osteoporosis; and (V) included as outcome indicators BMD, body mass index (BMI), and BMFF.

Excluded from the meta-analysis were: (I) repeated publications of data in the same group; (II) reviews, conference reports, personal experiences, case reports, and reviews; (III) studies unrelated to the subject of this study; (IV) studies with no research control group, or no comparability of samples between groups; and (V) studies in which the report of outcome indicators was not clear or the data used to support the results were incomplete.

\section{Quality assessment}

Two researchers independently reviewed the retrieved literature, reading the full-text and extracting relevant data from each article. Discrepancy between the two researchers was resolved by consensus, through discussion or by a third independent researcher, when required. The Jadad scale was used to evaluate the quality of the studies, based on: (I) categorization as randomized controlled studies; (II) appropriateness of the random method used; (III) application of the double-blind test; (IV) appropriateness of the double-blind method adopted; (V) loss of follow-up or withdrawal of patients in the process of the study, including whether the reasons were expounded and the analysis of intentionality was adopted. The scoring system allocated each "Yes" 1 point and each "No" 0 point, for a total of 5 points. A total score of less than 2 points was classified as low-grade research, while a score greater than 2 points was classified as high-grade research.

The Cochrane Reviewer's Handbook, v. 4.2.5, was used to evaluate the quality of the literature. The evaluation included consideration of whether: (I) the trial was randomized; (II) there was allocation concealment; (III) the blind test was used; (IV) the data used to support the results were complete; $(\mathrm{V})$ there was selective reporting of results; and (VI) there were other deviations.

\section{Data extraction}

Data extraction procedures were adopted, and the following data were extracted for each article: (I) general information about the article, including first author and publication year; (II) number of research participants, experimental design, specific measures used, study duration, and outcome indicators (which informed the evaluation of the results); (III) baseline data of patients; and (IV) feedback on research quality indicators.

\section{Statistical processing}

RevMan v. 5.3 software (The Cochrane Collaboration, Copenhagen, Denmark) was used to analyze the extracted data. First, the heterogeneity of the test results was tested, it mainly conducted homogeneity tests by $\chi^{2}$ inspection, significance level $\alpha=0.05$. The Peto method was used to analyze the heterogeneity $\left(\mathrm{I}^{2}\right)$ of the literature. If $\mathrm{P}>0.05$, $\mathrm{I}^{2}<50 \%$, it was considered that the included document has homogeneity or consistency, using a fixed effect model; if $\mathrm{P}<0.05, \mathrm{I}^{2}>50 \%$, it was considered that there is heterogeneity in the present data included, and the random effect model was used. The weighted mean difference (WMD) was used to represent the results of measurement data with the same measurement unit; otherwise, standard mean difference (SMD) was used to represent the results. The results of the enumerated data were expressed as relative risk (RR). All results were presented with a $95 \%$ confidence interval (CI). Funnel plots were drawn, and publication bias was evaluated based on the symmetry of funnel plots and the degree of literature concentration to the midline. Sensitivity analysis was used to evaluate the reliability and stability of the results.

\section{Results}

\section{Literature search results}

A total of 1,662 records were retrieved from the databases, and 1,447 abstracts were deemed relevant after deleting duplicates. Two researchers screened the titles of the articles and the abstracts, and then selected 125 articles that met the requirements for inclusion in the meta-analysis. After reading the full text of each article, 7 articles were identified that met the requirements for inclusion in the metaanalysis (13-19), excluding non-random, republished and inaccessible literature (Figure 1, Table 1). 
Identification of studies via databases and registers

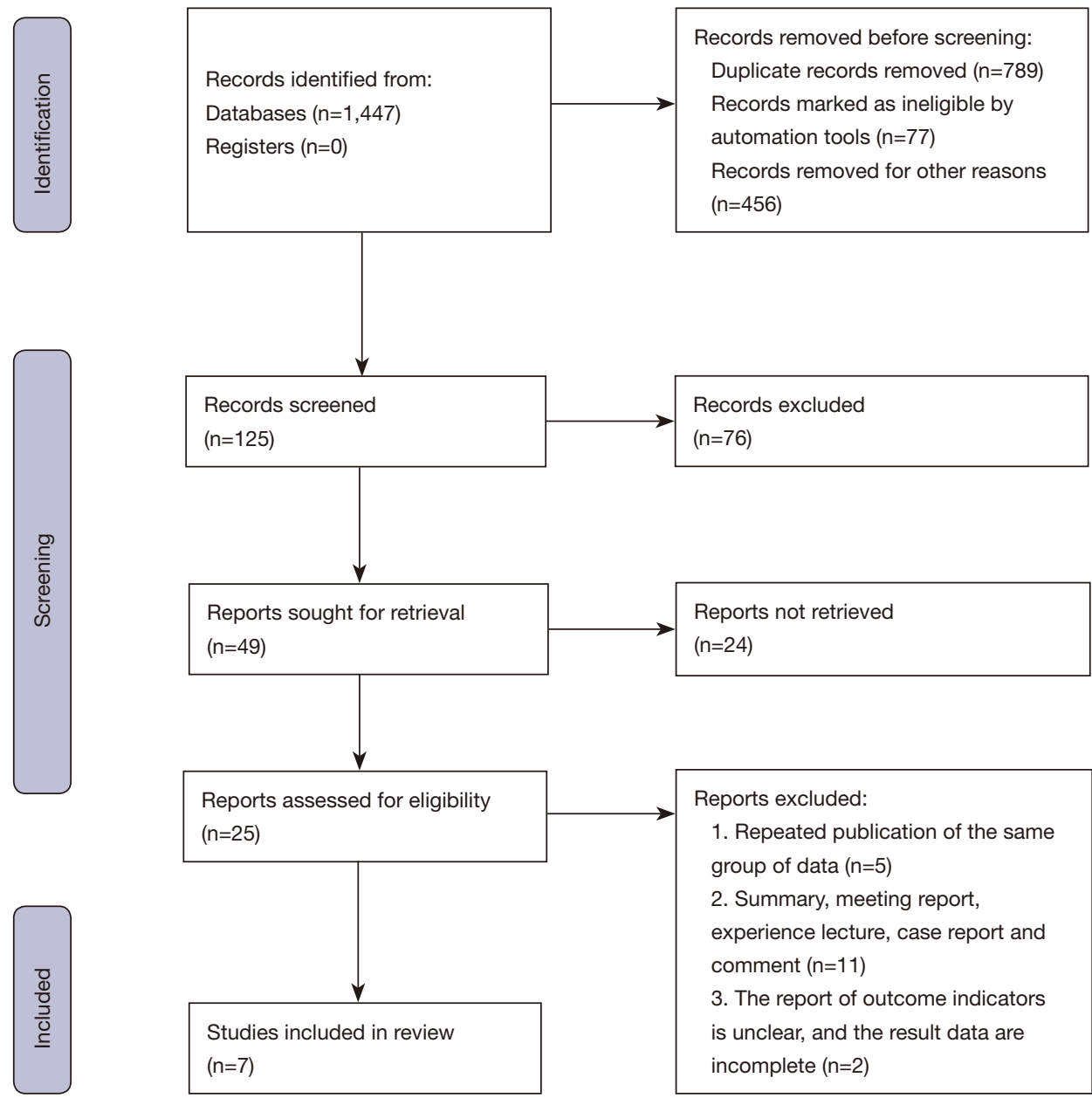

Figure 1 Literature retrieval process.

Table 1 Basic characteristics of the included literature

\begin{tabular}{lcccc}
\hline The first author & Year & Outcome indicators & Normal & Osteoporotic \\
\hline Yihao Guo & 2019 & BMD, BMI, BMFF & 26 & 92 \\
Jens-Peter & 2013 & BMD & 41 & 16 \\
Guanwu Li & 2016 & BMD, BMI & 135 & 11 \\
Yinxia Zhao & 2019 & BMD, BMI, BMFF & 39 & 13 \\
Guglielmo Manenti & 2013 & BMD, BMI, BMFF & 22 & 34 \\
James F. Griffith & 2008 & BMD, BMI & 25 & 11 \\
Jie He & 2017 & BMD, BMI, BMFF &
\end{tabular}

$\mathrm{BMD}$, bone mineral density; BMI, body mass index; BMFF, bone marrow fat fraction. 


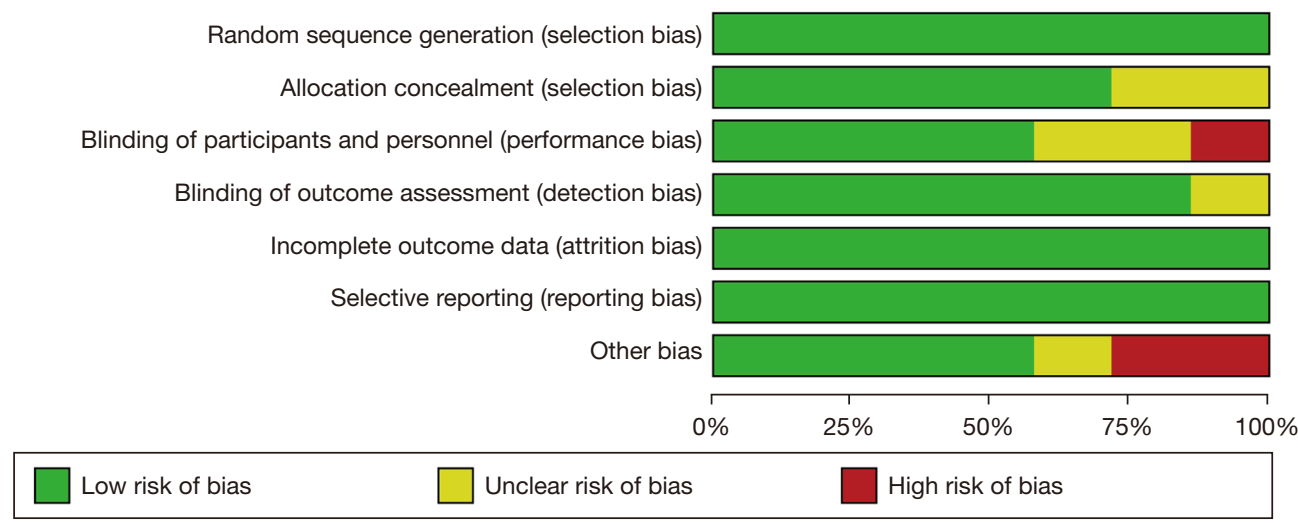

Figure 2 Bias evaluation of included articles.

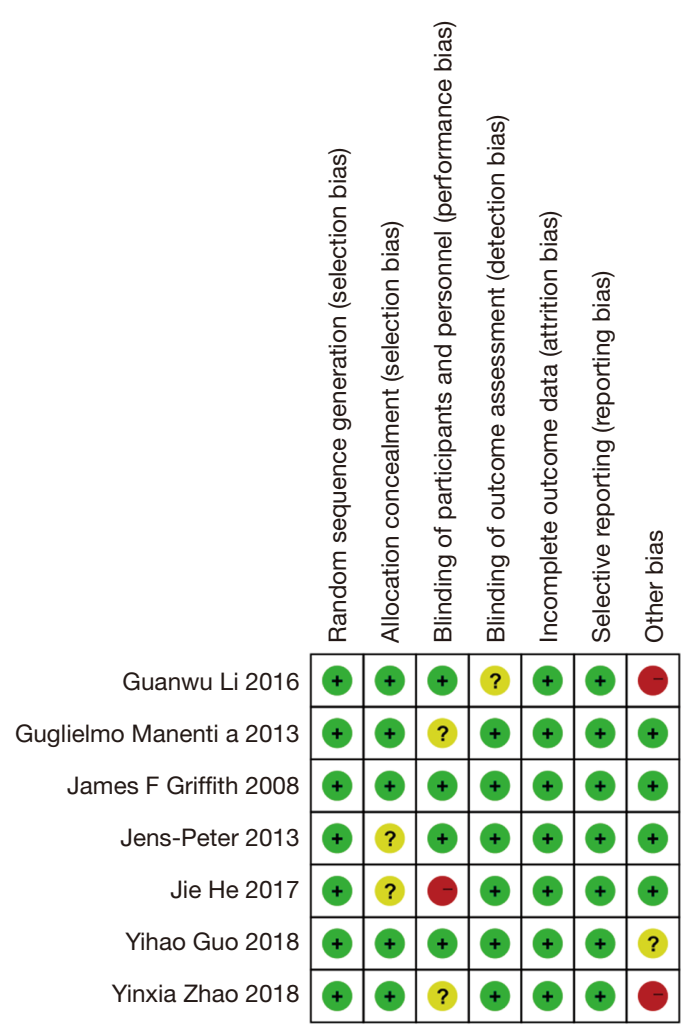

Figure 3 Bias-risk assessment of included articles.

\section{Bias-risk assessment of articles included in the meta- analysis}

First, the quality of the articles included in the metaanalysis was evaluated using the Cochrane Collaboration's Risk of Bias Tool; the results are shown in Figure 2 and Figure 3. There were no random sequence generation (selection bias), incomplete outcome data (selection bias), or selective reporting in all 7 studies (reporting bias). Overall, the risk included in this study was low (Figures 2,3).

The Jadad scale was used to evaluate the quality of the 7 articles included in the study (Table 2).

\section{Meta-analysis of BMD scores}

The BMD scores assessed by MRI chemical-shift imaging were compared between the normal group and the osteoporosis group. Heterogeneity was observed in the BMD scores between the normal group and the osteoporosis group ( $\left.\mathrm{I}^{2}=99 \% ; \mathrm{P}<0.00001\right)$, hence the random-effects model was used for the statistical analysis. The total BMD score of the normal group and the osteoporosis group was $M D=41.91$ (95\% CI: 40.15 to 43.66), with $Z=46.82$ $(\mathrm{P}<0.00001)$ (where the $Z$-score is the number of standard deviations above or below the mean for gender, ethnicity, and age-matched healthy population). The BMD scores of the two groups were statistically significant (Figure 4).

\section{Meta-analysis of BMI scores}

The BMI scores assessed by MRI chemical-shift imaging were compared between the normal group and the osteoporosis group. No heterogeneity was observed in the BMI scores between the normal group and the osteoporosis group $\left(\mathrm{I}^{2}=0 \%, \mathrm{P}=0.60\right)$, so a fixed-effect model was used for the statistical analysis. The total BMI score of patients in the normal group and osteoporosis group was $\mathrm{MD}=-1.43$ (95\% CI: -2.02 to -0.84$)$, with $\mathrm{Z}=4.76(\mathrm{P}<0.00001)$. There was a statistically significant difference in the BMI scores 
Table 2 Assessment of the quality of the literature using the Jadad scale

\begin{tabular}{|c|c|c|c|c|c|c|}
\hline The first author & Randomization & Binding & $\begin{array}{c}\text { Allocation } \\
\text { concealment }\end{array}$ & $\begin{array}{l}\text { Withdrawals and } \\
\text { dropouts }\end{array}$ & $\begin{array}{l}\text { Reason of dropouts } \\
\text { and withdrawals }\end{array}$ & Jadad \\
\hline Yihao Guo & Yes & No & NMT & MT & No & 3 \\
\hline Jens-Peter & Yes & No & NMT & MT & Yes & 3 \\
\hline Guanwu Li & Yes & No & NMT & MT & Yes & 3 \\
\hline James F. Griffith & Yes & No & NMT & MT & No & 3 \\
\hline Manenti & Yes & No & NMT & MT & No & 3 \\
\hline
\end{tabular}

NMT, not mentioned; MT, mentioned.

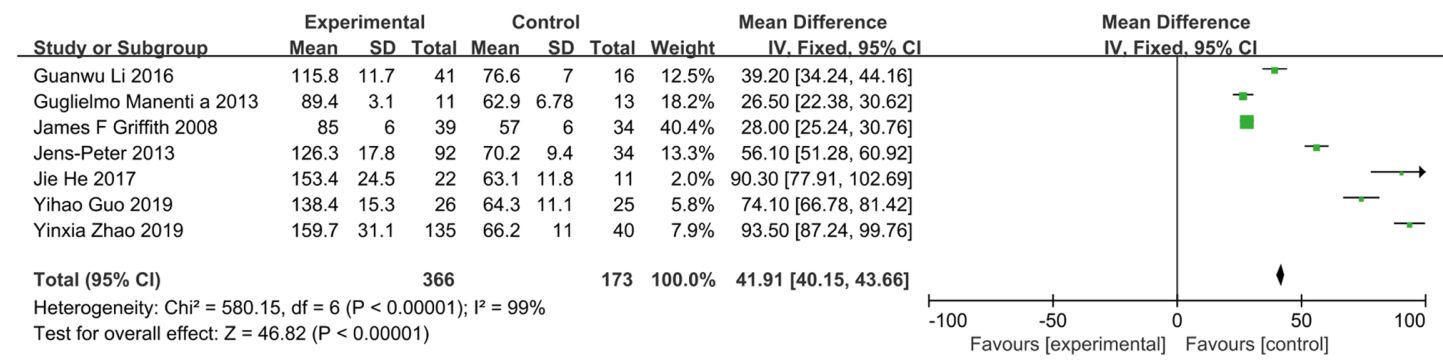

Figure 4 Forest plot of BMD scores assessed by MRI chemical-shift imaging. BMD, bone mineral density; MRI, magnetic resonance imaging.

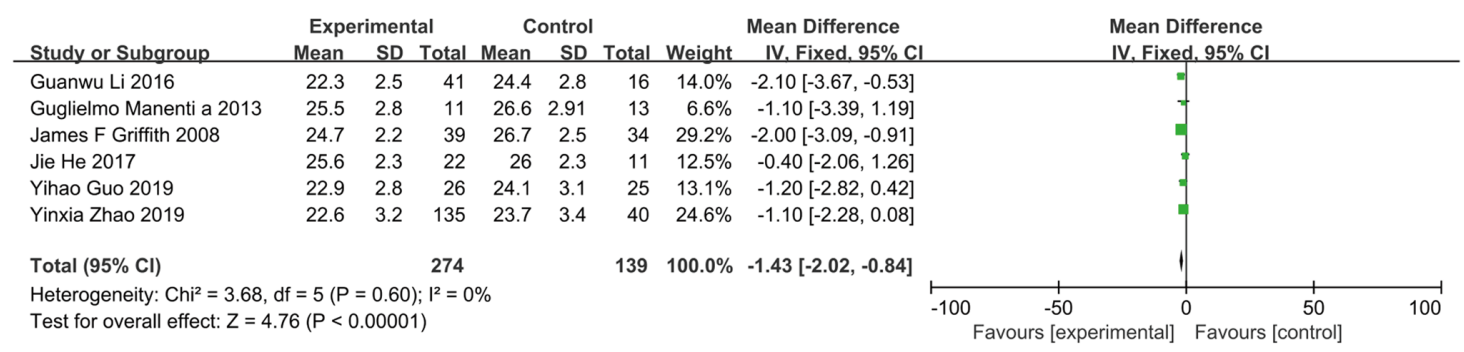

Figure 5 Forest plot of BMI scores assessed by MRI chemical-shift imaging. BMI, body mass index; MRI, magnetic resonance imaging.

between the two groups (Figure 5).

\section{Meta-analysis of BMFF scores}

The BMFF scores assessed by MRI chemical-shift imaging were compared between the normal group and the osteoporosis group. Heterogeneity was observed in the BMFF scores between the normal group and the osteoporosis group $\left(\mathrm{I}^{2}=79 \%, \mathrm{P}=0.003\right)$, so a random-effects model was used for the statistical analysis. The total BMFF score in the normal group and osteoporosis group was $\mathrm{MD}=-18.34$ (95\% CI: -20.32 to -16.36$)$, with $\mathrm{Z}=18.15$ $(\mathrm{P}<0.00001)$. The BMFF scores of the two groups were statistically significant (Figure 6).

\section{Publication bias analysis}

The publication bias of the BMD total score, the BMI 


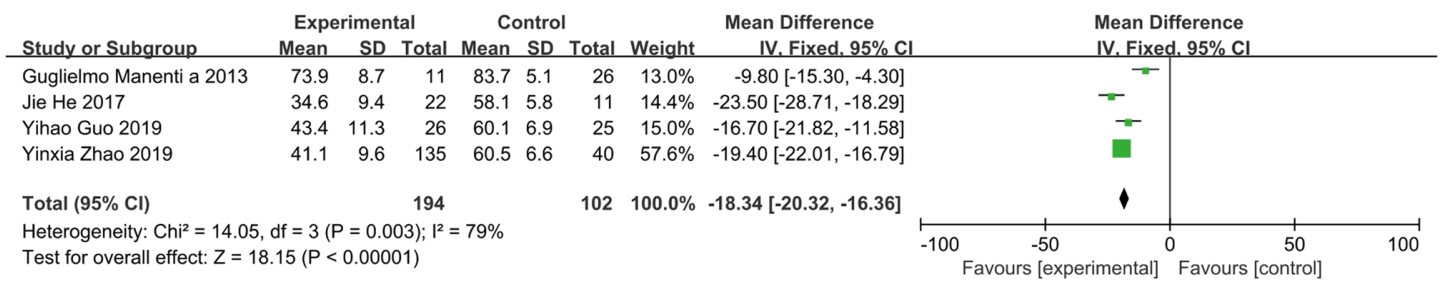

Figure 6 Forest plot of BMFF scores assessed by MRI chemical-shift imaging. BMFF, bone marrow fat fraction; MRI, magnetic resonance imaging.

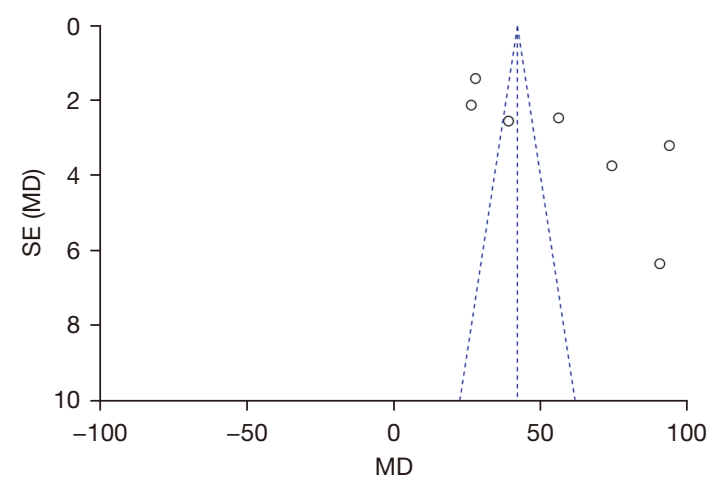

Figure 7 Funnel plot of BMD score. BMD, bone mineral density.

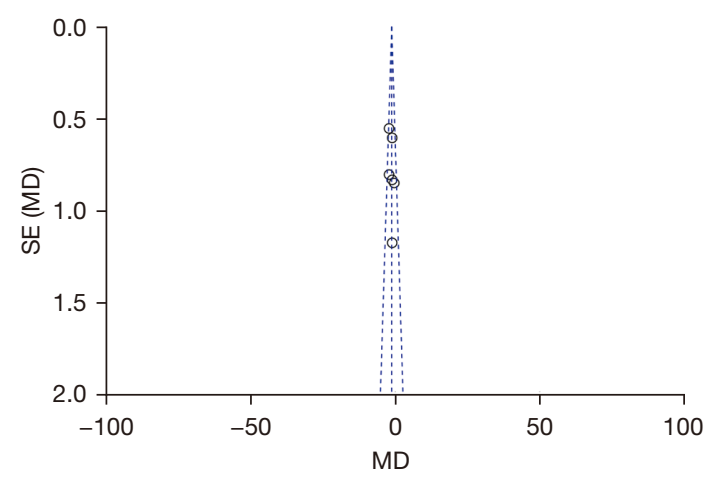

Figure 8 Funnel plot of BMI score. BMI, body mass index.

score, and the BMFF score (the three indexes evaluated by MRI chemical-shift imaging for patients in the normal group and osteoporosis group), were analyzed. The funnel plots of the BMD total score, the BMI score, and the BMFF score were relatively symmetric, and the data were relatively concentrated. As can be seen in Figure 7, only a few samples did not fall into the funnel plot. The results showed that the BMD total score, the BMI score and the BMFF score in this study had no significant publication bias (Figures 7-9).

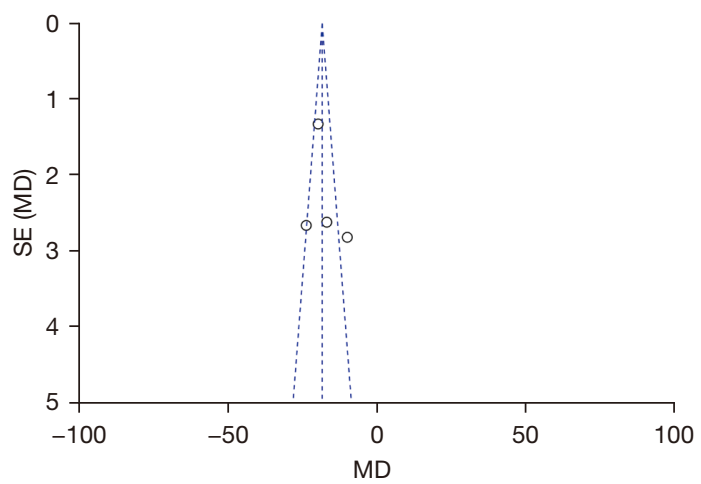

Figure 9 Funnel plot of BMFF score. BMFF, bone marrow fat fraction.

\section{Discussion}

Osteoporosis is a systemic metabolic bone disease with no obvious symptoms in its early stages. The decline of whole body BMD and bone mass are associated with the destruction of the body's bony microstructure, due to a variety of reasons. Osteoporosis can develop as a primary disorder (primary osteoporosis) or secondarily due to some other factor (secondary osteoporosis) (20). The etiology of primary osteoporosis is often uncertain, while the cause of secondary osteoporosis is usually relatively clear. Osteoporosis is generally caused by endocrine and metabolic diseases or hormonal factors affecting bone cell metabolism. Primary osteoporosis is generally classified into two categories: type I postmenopausal osteoporosis and type II senile osteoporosis $(21,22)$.

According to current data, about 200 million people are affected by osteoporosis, globally. In 2018, in China, 3.2\% of people aged from 40 to 49 years and $6 \%$ of men and $32.1 \%$ of women over 50 years suffered from osteoporosis. The prevalence of osteoporosis in women over 65 years old is as high as $51.6 \%(23,24)$. China is now an aging society, and it is predicted that by 2050 the disability rate caused 
by osteoporosis will be two to three times higher than it is today (25). The incidence of osteoporosis increases with age, due to the gradual decrease of osteoblasts. Osteoporosis is a major public health burden; therefore, finding ways to enhance the early diagnosis and prevention of osteoporosis in the elderly population is of paramount importance. DXA has long been regarded as the gold standard for the clinical evaluation of BMD and the diagnosis of osteoporosis. It has the advantages of fast detection time and low radiation dose and was once the preferred method for measuring BMD. However, data also show that DXA has low accuracy and a high rate of missed diagnosis. Furthermore, because MRI signals from solid-state tissues such as bone are very low and do not show details of bone very well, MRI was not applied to the measurement of BMD in clinical practice (26-28). Studies have shown, though, that MRI chemicalshift imaging can be very good at performing bone density measurements, by measuring the cone signal decline index to reflect the change of bone fat content, thus proving the value of MRI chemical-shift imaging in the diagnosis and evaluation of osteoporosis (29,30).

In addition, advances in MRI hardware and software technology and the increased use of DWI are slowly making it possible to index and classify osteoporosis. By studying the ADC values in patients with lumbar vertebral body measurements, vertebral osteoporosis in patients can be evaluated to support early detection and prevention and, to a certain extent, save medical costs associated with a delayed diagnosis.

To sum up, MRI chemical-shift imaging technology is an excellent imaging detection method. It has an inspection sequence that uses the phenomenon of the chemical shift of protons at the resonance frequency of tissue compounds to determine the composition of body tissues. Using MRI chemical-shift imaging can perform high-precision measurement of fat content in bones and its proportion of water, expressed bone fat content in cell molecules, can detect bone density and bone marrow physiological pathological changes of osteoporosis patients. However, the number of samples constrained in the study should be less insufficient, and other test techniques should be used to expand the quality of bone quality in combination, and further theoretical basis for the quality assessment of osteoporosis is further approved.

\section{Conclusions}

This systematic literature review and meta-analysis examined the value of MRI chemical-shift imaging in assessing bone quality in patients with osteoporosis. A total of 7 relevant studies were identified and included in the analysis. The BMD, BMI and BMFF scores, as assessed by MRI chemical-shift imaging, were compared between the normal group and the osteoporosis group. For patients with osteoporosis, the following scores were observed: BMD: $M D=41.91$ (95\% CI: 40.15 to 43.66 ), $Z=46.82$ $(\mathrm{P}<0.00001)$; BMI: $\mathrm{MD}=-1.43$ (95\% CI: -2.02 to -0.84$)$, $\mathrm{Z}=4.76(\mathrm{P}<0.00001)$; BMFF: $\mathrm{MD}=-18.34$ (95\% CI: -20.32 to -16.36$), \mathrm{Z}=18.15(\mathrm{P}<0.00001)$.

These results indicate that MRI chemical-shift imaging can effectively evaluate BMD and bone mass in patients with osteoporosis. However, given that the only outcome indicators included in this analysis were BMD, BMI, and $\mathrm{BMFF}$, it is necessary to expand the sample size and outcome indicators in future research. According to the results of this analysis, MRI chemical-shift imaging can effectively measure and evaluate BMD and bone mass, including in patients with osteoporosis.

\section{Acknowledgments}

Funding: This study was supported by the Research level of Affiliated Hospital of North Sichuan Medical University, 2020JC029.

\section{Footnote}

Reporting Checklist: The authors have completed the PRISMA reporting checklist. Available at https://dx.doi. org/10.21037/apm-21-3479

Conflicts of Interest: All authors have completed the ICMJE uniform disclosure form (available at https://dx.doi. org/10.21037/apm-21-3479). The authors have no conflicts of interest to declare.

Ethical Statement: The authors are accountable for all aspects of the work in ensuring that questions related to the accuracy or integrity of any part of the work are appropriately investigated and resolved.

Open Access Statement: This is an Open Access article distributed in accordance with the Creative Commons Attribution-NonCommercial-NoDerivs 4.0 International License (CC BY-NC-ND 4.0), which permits the noncommercial replication and distribution of the article with 
the strict proviso that no changes or edits are made and the original work is properly cited (including links to both the formal publication through the relevant DOI and the license). See: https://creativecommons.org/licenses/by-nc-nd/4.0/.

\section{References}

1. Johnston CB, Dagar M. Osteoporosis in Older Adults. Med Clin North Am 2020;104:873-84.

2. Ensrud KE, Crandall CJ. Osteoporosis. Ann Intern Med 2017;167:ITC17-32.

3. Rizzoli R. Postmenopausal osteoporosis: Assessment and management. Best Pract Res Clin Endocrinol Metab 2018;32:739-57.

4. Chen $\mathrm{P}, \mathrm{Li} \mathrm{Z}, \mathrm{Hu}$ Y. Prevalence of osteoporosis in China: a meta-analysis and systematic review. BMC Public Health 2016;16:1039.

5. Yu F, Xia W. The epidemiology of osteoporosis, associated fragility fractures, and management gap in China. Arch Osteoporos 2019;14:32.

6. Głogowska-Szeląg J. Assessment of the relationship between bmd and body mass index bmi in women with postmenopausal osteoporosis. Wiad Lek 2018;71:1714-8.

7. Jung EY, Park SJ, Shim HE, et al. Multidisciplinary team training reduces the error rate of DXA image. Arch Osteoporos 2020;15:115.

8. Zeng Q, Li N, Wang Q, et al. The Prevalence of Osteoporosis in China, a Nationwide, Multicenter DXA Survey. J Bone Miner Res 2019;34:1789-97.

9. Löffler MT, Sollmann N, Mei K, et al. X-ray-based quantitative osteoporosis imaging at the spine. Osteoporos Int 2020;31:233-50.

10. Momeni M, Asadzadeh M, Mowla K, et al. Sensitivity and specificity assessment of DWI and ADC for the diagnosis of osteoporosis in postmenopausal patients. Radiol Med 2020;125:68-74.

11. Tang GY, Lv ZW, Tang RB, et al. Evaluation of MR spectroscopy and diffusion-weighted MRI in detecting bone marrow changes in postmenopausal women with osteoporosis. Clin Radiol 2010;65:377-81.

12. Allon R, Levy Y, Lavi I, et al. How to Best Predict Fragility Fractures: An Update and Systematic Review. Isr Med Assoc J 2018;20:773-9.

13. Guo Y, Chen Y, Zhang X, et al. Magnetic Susceptibility and Fat Content in the Lumbar Spine of Postmenopausal Women With Varying Bone Mineral Density. J Magn Reson Imaging 2019;49:1020-8.

14. Kühn JP, Hernando D, Meffert PJ, et al. Proton-density fat fraction and simultaneous R2* estimation as an MRI tool for assessment of osteoporosis. Eur Radiol 2013;23:3432-9.

15. Li G, Xu Z, Gu H, et al. Comparison of chemical shift-encoded water-fat MRI and MR spectroscopy in quantification of marrow fat in postmenopausal females. J Magn Reson Imaging 2017;45:66-73.

16. Zhao Y, Huang M, Ding J, et al. Prediction of Abnormal Bone Density and Osteoporosis From Lumbar Spine MR Using Modified Dixon Quant in 257 Subjects With Quantitative Computed Tomography as Reference. J Magn Reson Imaging 2019;49:390-9.

17. Manenti G, Capuani S, Fanucci E, et al. Diffusion tensor imaging and magnetic resonance spectroscopy assessment of cancellous bone quality in femoral neck of healthy, osteopenic and osteoporotic subjects at 3T: Preliminary experience. Bone 2013;55:7-15.

18. Griffith JF, Yeung DK, Tsang PH, et al. Compromised bone marrow perfusion in osteoporosis. J Bone Miner Res 2008;23:1068-75.

19. He J, Fang H, Li X. Vertebral bone marrow fat content in normal adults with varying bone densities at $3 \mathrm{~T}$ magnetic resonance imaging. Acta Radiol 2019;60:509-15.

20. Danford CJ, Trivedi HD, Papamichael K, et al. Osteoporosis in primary biliary cholangitis. World J Gastroenterol 2018;24:3513-20.

21. Arundel P, Bishop N. Primary Osteoporosis. Endocr Dev 2015;28:162-75.

22. Lorentzon M, Cummings SR. Osteoporosis: the evolution of a diagnosis. J Intern Med 2015;277:650-61.

23. Ballane G, Cauley JA, Luckey MM, et al. Worldwide prevalence and incidence of osteoporotic vertebral fractures. Osteoporos Int 2017;28:1531-42.

24. Gao C, Xu Y, Li L, et al. Prevalence of osteoporotic vertebral fracture among community-dwelling elderly in Shanghai. Chin Med J (Engl) 2019;132:1749-51.

25. Catalano A, Martino G, Morabito N, et al. Pain in Osteoporosis: From Pathophysiology to Therapeutic Approach. Drugs Aging 2017;34:755-65.

26. Clotet J, Martelli Y, Di Gregorio S, et al. Structural Parameters of the Proximal Femur by 3-Dimensional Dual-Energy X-ray Absorptiometry Software: Comparison With Quantitative Computed Tomography. J Clin Densitom 2018;21:550-62.

27. Lopez Picazo M, Magallon Baro A, Del Rio Barquero LM, et al. 3-D Subject-Specific Shape and Density Estimation of the Lumbar Spine From a Single Anteroposterior DXA Image Including Assessment of Cortical and Trabecular 
Bone. IEEE Trans Med Imaging 2018;37:2651-62.

28. Chang G, Boone S, Martel D, et al. MRI assessment of bone structure and microarchitecture. J Magn Reson Imaging 2017;46:323-37.

29. Sollmann N, Löffler MT, Kronthaler S, et al. MRI-Based Quantitative Osteoporosis Imaging at the Spine and Femur. J Magn Reson Imaging 2021;54:12-35.

Cite this article as: Wang Y, Li H, Yang X, Zhang C, Xie H. Systematic review and meta-analysis: the value of MRI chemical-shift imaging in the evaluation of bone quality in patients with osteoporosis. Ann Palliat Med 2021;10(12):1270612715. doi: 10.21037/apm-21-3479
30. Martel D, Leporq B, Saxena A, et al. 3T chemical shiftencoded MRI: Detection of altered proximal femur marrow adipose tissue composition in glucocorticoid users and validation with magnetic resonance spectroscopy. J Magn Reson Imaging 2019;50:490-6.

(English Language Editor: K. Gilbert) 